\title{
Myelin oligodendrocyte glycoprotein antibody encephalitis following severe acute respiratory syndrome coronavirus 2 in a pediatric patient
}

An otherwise healthy, 7-year-old female presented acutely with status epilepticus, aphasia, fever, encephalopathy, and prolonged Todd's paralysis. The week prior to admission, the patient reported 2 self-limited generalized convulsions which did not require acute care. Labs on admission are displayed in Table 1. The patient's neuroimaging (Fig. 1) revealed peri Rolandic and posterior parietal lobe restricted diffusion and cortical edema. Her electroencephalogram was abnormal as well, demonstrating cerebral slowing with left focal slowing. Of note, severe acute respiratory syndrome coronavirus 2 (SARS-

Table 1. Pertinent lab findings from hospital admissions

\begin{tabular}{|c|c|c|}
\hline Laboratory finding & Hospital admission 1 & $\begin{array}{l}\text { Hospital admission } 2 \\
\text { (+7 days) }\end{array}$ \\
\hline \multicolumn{3}{|l|}{ Serum } \\
\hline MOG IgG & $1: 40$ & $1: 100$ \\
\hline SARS-CoV-2 PCR & Negative & Negative \\
\hline SARS-CoV-2 IgG & Not obtained & Positive (titer: 7.1) \\
\hline Aquaporin-4 antibody & Negative & Negative \\
\hline TNF-a & Not obtained & $1.68 \mathrm{pg} / \mathrm{mL}(\mathrm{H})$ \\
\hline \multicolumn{3}{|l|}{ CSF } \\
\hline White blood cell & $132 \mathrm{cell} / \mathrm{mm}^{3}$ & 116 cell $/ \mathrm{mm}^{3}$ \\
\hline$\%$ lymphocytes & $64 \%$ & $90 \%$ \\
\hline Red blood cell & 1,000 cell $/ \mathrm{mm}^{3}$ & 0 cell $/ \mathrm{mm}^{3}$ \\
\hline Total protein & $51 \mathrm{mg} / \mathrm{dL}$ & 48 mg/dL \\
\hline Glucose & 73 mg/dL & 46 mg/dL \\
\hline Film arraya & Negative & Negative \\
\hline Mayo encephalitis panel & Negative & Negative \\
\hline Oligoclonal bands & Not obtained & $\begin{array}{l}\text { Multiple OCB present } \\
\text { in CSF/serum }\end{array}$ \\
\hline IgG index & Not obtained & 0.55 \\
\hline
\end{tabular}

Film array is an institution specific polymerase chain reaction for 14 pathogens including Escherichia coli, Haemophilus influenzae, Listeria, Neisseria meningitidis, strep agalactiae, strep pneumonia, cytomegalovirus, enterovirus, herpes simplex virus (HSV) $1 / 2$, human herpesvirus 6, human Par echovirus, varicella zoster virus, Cryptococcus neoformans. Comprehensive meningoencephalitis panel including Epstein-Barr virus, HSV 1/2, West Nile Virus, Western Equine Encephalitis, St. Louis Encephalitis, California Encephalitis, Eastern Equine Encephalitis, lymphocytic choriomeningitis virus, mumps virus with additional spectrum testing for mycoplasma pneumoniae, tuberculosis, rickettsia, and bartonella was negative.

MOG, myelin oligodendrocyte glycoprotein; SARS-CoV-2 PCR, severe acute respiratory syndrome coronavirus 2 polymerase chain reaction; TNF-a, tumor necrosis factor alpha; OCB, Oligoclonal bands; CSF, cerebrospinal fluid.
CoV-2) polymerase chain reaction was negative in the serum and cerebrospinal fluid although immunoglobulin $G$ levels were not obtained. Following intervention with anticonvulsant therapy, she returned to her neurocognitive baseline and was discharged 2 days after admission.

The patient was readmitted the following week in with headaches, encephalopathy, abdominal pain, dysarthria/slurred speech, and altered mental status. Repeat magnetic resonance imaging brain showed previous diffusion restriction although cortical edema was less apparent. Labs are displayed in Table 1 but on this admission, SARS-CoV-2 antibodies were checked, revealing a titer of 7.1. The patient was also found to be myelin oligodendrocyte glycoprotein (MOG) antibody positive with a titer of 1:100. For presumed postinfectious neurologic phenomenon, the patient was administered intravenous immunoglobulin $2 \mathrm{~g} / \mathrm{kg}$ over 3 days. She showed improvement in her condition over 5 days and was discharged home with no further seizures. Upon follow-up, she had almost returned to her baseline with mild dysarthria.

\section{Neurologic complications associated with SARS-CoV-2}

Neurologic complications associated with SARS-CoV-2 have been reported with retrospective studies identifying between $14 \%-67 \%$ patients experience neurological disease during the acute infectious period. ${ }^{1)}$ Reported neurologic phenomenon include anosmia, ageusia, seizures, meningoencephalitis, cerebrovascular disease, Guillain-Barré syndrome, postviral polyneuropathies, encephalitis, and acute disseminated encephalomyelitis (ADEM) particularly with hemorrhage (also termed acute necrotizing encephalitis). ${ }^{2}$ Roughly $34 \%$ of children with SARSCoV-2 related multisystem inflammatory syndrome in children have identified neurological manifestations, although these have generally been less severe phenotypes than observed in adults. ${ }^{3)}$ Although encephalitis has been reported, this was in the setting of acute infection, ${ }^{4}$ and thus far no postinfectious neurologic phenomenon have been reported. 


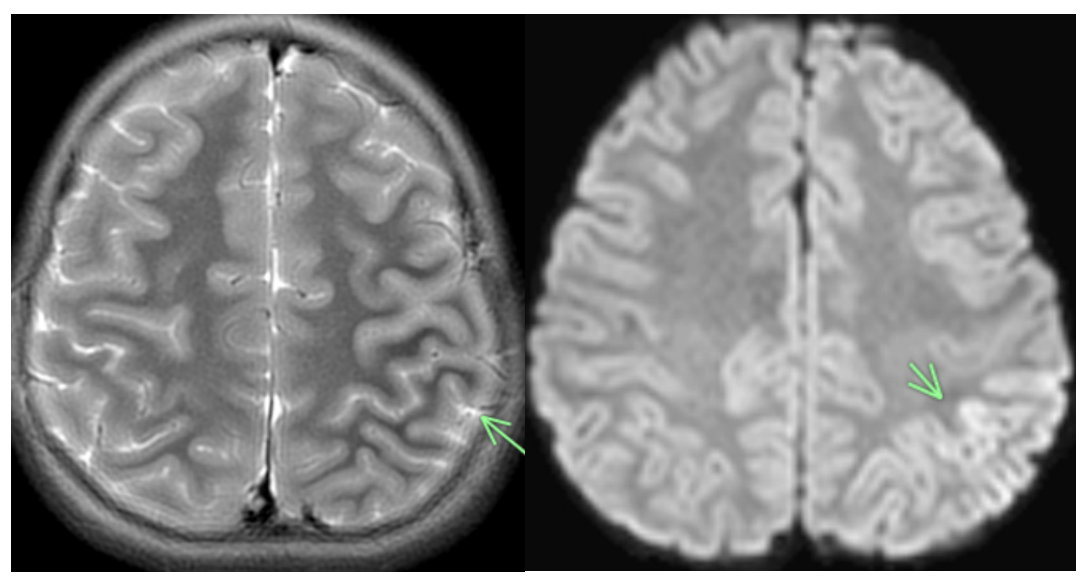

Fig. 1. (A) Axial T2 showing Left perirolandic cortex and posterior parietal lobe cerebral edema marked by the arrow. (B) Axial diffusion weighted image showing restricted diffusion marked by the arrow.

\section{Myelin oligodendrocyte glycoprotein (MOG) antibodies in children}

In children, MOG antibodies are associated with a variety of postinfectious acquired demyelinating disorders, demonstrating a strong predilection for children. MOG antibodies have been identified over the last decade to be intimately linked with acquired demyelinating diseases with a strong predilection for striking in the pediatric age spectrum. Reported neurologic phenomenon associated with MOG antibodies include ADEM, optic neuritis, transverse myelitis, neuromyelitis optica, and autoimmune encephalitis. Although the exact etiology to MOG spectrum disorders is unknown, postinfectious molecular mimicry and epitope spreading are hypothesized. MOG antibody associated encephalomyelitis has been reported in $67 \%$ with infectious prodrome and have been related to postinfectious conditions including herpes simplex virus (HSV), Epstein-Barr virus (EBV), others. ${ }^{5)}$ Armangue et al. ${ }^{6}$ have shown in their prospective study that $\mathrm{HSV}$ encephalitis can trigger autoimmune encephalitis in $27 \%$ of patients. SARS-CoV-2 being neurotropic might play similar role.

\section{MOG and SARS-CoV-2}

To date, there have been no associated cases of MOG spec. trum disorders in children associated with either acute or postinfectious infection with SARS-CoV-2. Here we present a case of a 7-year-old female with a prior asymptomatic infection with SARS-CoV-2 who subsequently developed encephalopathy and status epilepticus in association with MOG antibody positivity. The presence of SARS-CoV-2 could be a coincidence, but a prior study has shown higher rates of nonencephalitic past HSV-1 infection significantly more in N-methyl-D-aspartate receptor encephalitis suggesting meaningful association between infection and the antibody mediated encephalitis. ${ }^{7)}$ The phenotype in our case is characteristic of pediatric onset MOG spectrum dis- orders in that seizures, encephalopathy, and immunotherapy responsiveness are all reported. Although the patient's radiographic findings are inconsistent with ADEM the clinical presentation of encephalopathy and seizure does match this disorder well, as does the presence of MOG antibodies. For this reason, the authors feel strongly that the patients presentation is associated with the presence of SARS-CoV-2 antibodies in spite of the patient having no occult respiratory illness reported by the family.

\section{Mechanism of SARS-CoV-2 and autoimmune encephalitis}

The exact mechanism is obscure whereas SARS-CoV-2 had been associated with autoimmune encephalitis in pediatric, ${ }^{8)}$ adults and the proposed mechanism of MOG antibodies presence is likely heterogeneous including molecular mimicry with identification of self antigens as foreign in the presence of similarity of epitopes, or initial infection leading to dispersion of central nervous system antigens to periphery with development of autoantibodies to myelin protein. Although restricted diffusion was present in our case, the radiographic findings are inconsistent with cerebrovascular disease or vasculopathy although the latter has been reported in persons with MOG antibodies and SARS-CoV-2 infection. ${ }^{9)}$

\section{Two important factors to neurologist}

While singular cases are difficult to generalize to the greater population, there are 2 important factors of relevance to treating neurologists. Firstly, the potential link between prior SARSCoV-2 infection and the subsequent development of neuroinflammatory disease is of importance given the high rates of infection around the globe. Although prevention of SARS$\mathrm{CoV}-2$ is of primary importance, understanding that MOG 
antibody spectrum disorders can occur in the postinfectious period is of importance. Further, amongst neurologists and pediatricians evaluating patients with new onset seizure activity or ADEM-like presentations, ascertainment of SARS-CoV-2 antibody status may be of importance from both a treatment and prognostication standpoint as postinfectious neuroinflammation tends to be monophasic. ${ }^{10)}$ This is of greater importance in pediatric patients as there may be no occult respiratory phenomenon with initial infection.

\section{Key Message}

Question: Although neurologic complications have been reported during the acute phase of infection in children, less is known about the postinfectious phenomenon associated with the severe acute respiratory syndrome coronavirus 2 (SARSCoV-2) virus.

Finding: We present a case of a 7-year-old female with a prior asymptomatic infection with SARS-CoV-2 who subsequently developed encephalopathy and status epilepticus in association with myelin oligodendrocyte glycoprotein (MOG) antibody positivity.

Meaning: Here we present the first case of postinfectious of a MOG spectrum disorder associated with prior SARS-CoV-2 infection in a pediatric patient.

\section{Conflicts of interest}

No potential conflict of interest relevant to this article was reported.

Nusrat Ahsan, MD ${ }^{1,2}$, Saba Jafarpour ${ }^{1}$, Jonathan D. Santoro, $\mathrm{MD}^{1,2}$

${ }^{1}$ Division of Neurology, Department of Pediatrics, Children's Hospital Los Angeles, Los Angeles, CA, USA; ${ }^{2}$ Department of Neurology, Keck School of Medicine at the University of Southern California, Los Angeles, CA, USA

Corresponding author: Nusrat Ahsan, MD

Division of Neurology, Department of Pediatrics, Children's Hospital Los Angeles, 4650 Sunset Blvd, MS82, Los Angeles, CA 90027, USA

凶Email:nahsan@chla.usc.edu

https://orcid.org/0000-0002-3502-8658

\section{References}

1. Helms J, Kremer S, Merdji H, Clere-Jehl R, Schenck M, Kummerlen C, et al. Neurologic features in severe SARS-CoV-2 infection. N Engl J Med 2020;382:2268-70.

2. Ellul MA, Benjamin L, Singh B, Lant S, Michael BD, Easton A, et al. Neurological associations of COVID-19. Lancet Neurol 2020;19:76783.

3. Feldstein LR, Rose EB, Horwitz SM, Collins JP, Newhams MM, Son MBF, et al. Multisystem inflammatory syndrome in U.S. children and adolescents. NEngl J Med 2020;383:334-6.

4. McAbee GN, Brosgol Y, Pavlakis S, Agha R, Gaffoor M. Encephalitis associated with COVID-19 infection in an 11-year-old child. Pediatr Neurol 2020;109:94.

5. Hacohen Y, Absoud M, Deiva K, Hemingway C, Nytrova P, Woodhall M, et al. Myelin oligodendrocyte glycoprotein antibodies are associated with a non-MS course in children. Neurol Neuroimmunol Neuroinflamm 2015;2:e81.

6. Armangue T, Spatola M, Vlagea A, Mattozzi S, Cárceles-Cordon M, Martinez-Heras E, et al. Frequency, symptoms, risk factors, and outcomes of autoimmune encephalitis after herpes simplex encephalitis: a prospective observational study and retrospective analysis. Lancet Neurol 2018;17:760-72.

7. Salovin A, Glanzman J, Roslin K, Armangue T, Lynch DR, Panzer JA. Anti-NMDA receptor encephalitis and nonencephalitic HSV-1 infection. Neurol Neuroimmunol Neuroinflamm 2018;5:e458.

8. Burr T, Barton C, Doll E, Lakhotia A, Sweeney M. N-methyl-d-aspartate receptor encephalitis associated with COVID-19 infection in a toddler. Pediatr Neurol 2021;114:75-6.

9. Pinto AA, Carroll LS, Nar V, Varatharaj A, Galea I. CNS inflammatory vasculopathy with antimyelin oligodendrocyte glycoprotein antibodies in COVID-19. Neurol Neuroimmunol Neuroinflamm 2020;7:e813.

10. Hacohen Y, Wong YY, Lechner C, Jurynczyk M, Wright S, Konuskan B, et al. Disease course and treatment responses in children with relapsing myelin oligodendrocyte glycoprotein antibody-associated disease. JAMA Neurol 2018;75:478-87.

How to cite this article: Nusrat Ahsan, Saba Jafarpour, Jonathan D. Santoro. Myelin oligodendrocyte glycoprotein antibody encephalitis following severe acute respiratory syndrome coronavirus 2 in a pediatric patient. Clin Exp Pediatr 2021;64:310-2. https://doi.org/10.3345/cep.2020.01963 